\title{
Radial pulsation stability as a function of hydrogen abundance
}

\author{
C.S. Jeffery ${ }^{1}$ and H. Saio ${ }^{2}$ \\ 1 Armagh Observatory, College Hill, Armagh BT61 9DG, UK \\ ${ }^{2}$ Astronomical Institute, School of Science, Tohoku University, Sendai 980-8578, Japan
}

\begin{abstract}
We explore the radial (p-mode) stability of stars across a wide range of mass $(0.2<$ $\left.M<50 \mathrm{M}_{\odot}\right)$, composition $(0<X<0.7, Z=0.001,0.02)$, effective temperature, and luminosity. We identify the instability boundaries associated with low- to high-order radial oscillations $(0 \leqslant n \leqslant 13)$. The instability boundaries are a strong function of both composition and radial order $(n)$. The classical blue edge shifts to higher effective temperature and luminosity with decreasing hydrogen abundance. High-order modes are more easily excited and small islands of high radial-order instability develop, some of which correspond with real stars. Driving in all cases is by the classical $\kappa$-mechanism and, at high luminosity-to-mass ratio, strange-mode instability. We identify regions of parameter space where new classes of pulsating variable have recently or may, in future, be discovered. The majority of these are associated with reduced hydrogen abundance in the envelope.
\end{abstract}

Keywords. stars: oscillations, stars: interiors, stars: white-dwarf, stars: subdwarfs, stars: earlytype

\section{Introduction}

Since the discovery of periodic light variations in the luminous giant $\delta$ Cephei, the study of stellar pulsations has transformed our understanding of how stars work, as well as establishing a distance scale whereby the cosmos can be measured. The fact that the light variations in $\delta$ Cep represented a major discovery testifies to the fact that not all stars are variable. However, as telescopes and detectors have become more sensitive, pulsations have been identified in diverse groups of stars of all masses and across the Hertzsprung-Russell diagram. Such discoveries continue to the present day, with pulsations in low-mass white dwarfs and pre-white dwarfs being the latest additions to the pulsating star zoo (Maxted et al. 2013; Hermes et al. 2013).

Jeffery \& Saio (2013) demonstrated that pulsation instability in the low-mass prewhite dwarf J0247-25B would arise in a high-order overtone if the envelope was depleted in hydrogen. The principal reason for this, shown previously by Saio \& Jeffery (1988); Jeffery \& Saio $(2007,2013)$, is that hydrogen acts as a poison, diluting the steep positive opacity gradients $d \kappa / d T$ which arise on the low-temperature sides of major ionisation zones and which are the major drivers of instability if located at an appropriate depth beneath the stellar surface. When hydrogen is depleted or absent, the destabilizing effect of these ionization zones is increased.

The question is now whether it is possible to predict the properties of other hitherto undiscovered pulsating variables, especially those in which hydrogen has been depleted as a consequence of prior evolution. We pesent preliminary results in a new parametric survey to identify locations where new classes of variable star await discovery. 

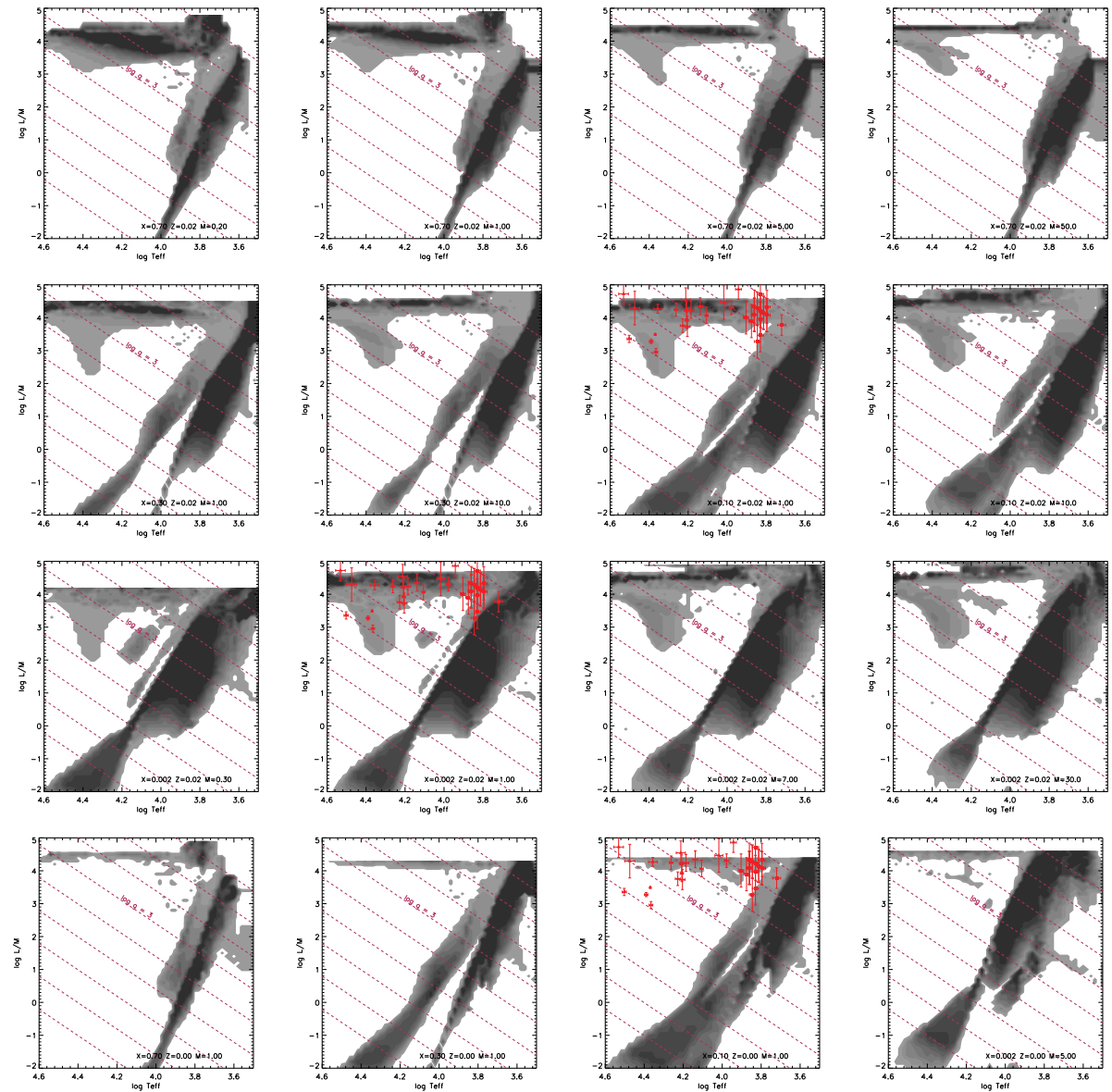

Figure 1. Unstable pulsation modes in stars with homogeneous envelopes for selected compositions and masses, as labelled. The number of unstable radial modes is represented by grey scale contours, with the lightest shade marking the instability boundary (one unstable mode), and the darkest shade representing ten or more more unstable modes. Broken (maroon) diagonal lines represent contours of constant surface gravity at $\log g=8,7,6, \ldots, 1$. Red symbols with error bars represent the observed positions of pulsating low-mass hydrogen-deficient stars, including extreme helium stars and R Coronae Borealis variables (Jeffery 2008), shown on panels with $X \leqslant 0.1$ and $M=1.0 \mathrm{M}_{\odot}$

\section{Radial Pulsation Models}

The pulsation stability survey comprises a grid of model envelopes covering: chemical mixtures $X=0.002,0.1,0.30,0.70$ and $Z=0.001,0.02$, masses $0.2 \leqslant M / \mathrm{M}_{\odot} \leqslant$ 50 , effective temperatures $\log T_{\text {eff }} / \mathrm{K}=3.50(0.02) 4.60$, and luminosity-to-mass ratios $\log \left(L / \mathrm{L}_{\odot}\right) /\left(M / \mathrm{M}_{\odot}\right)=-2.0(0.2) 5.0$.

The linear nonadiabatic analysis of stability against pulsation was carried out following methods described by Saio et al. (1983) and Jeffery \& Saio (2006a,b). For each model, the first 17 eigenfrequencies were located and stored, including real and imaginary components $\omega_{\mathrm{r}}$ and $\omega_{\mathrm{i}}$, the period $\Pi$ and the number of nodes in the eigensolution. Modes with $\omega_{\mathrm{i}}<0$ were deemed to be unstable because the temporal dependence was assumed to be $\exp (i \omega t)$. 

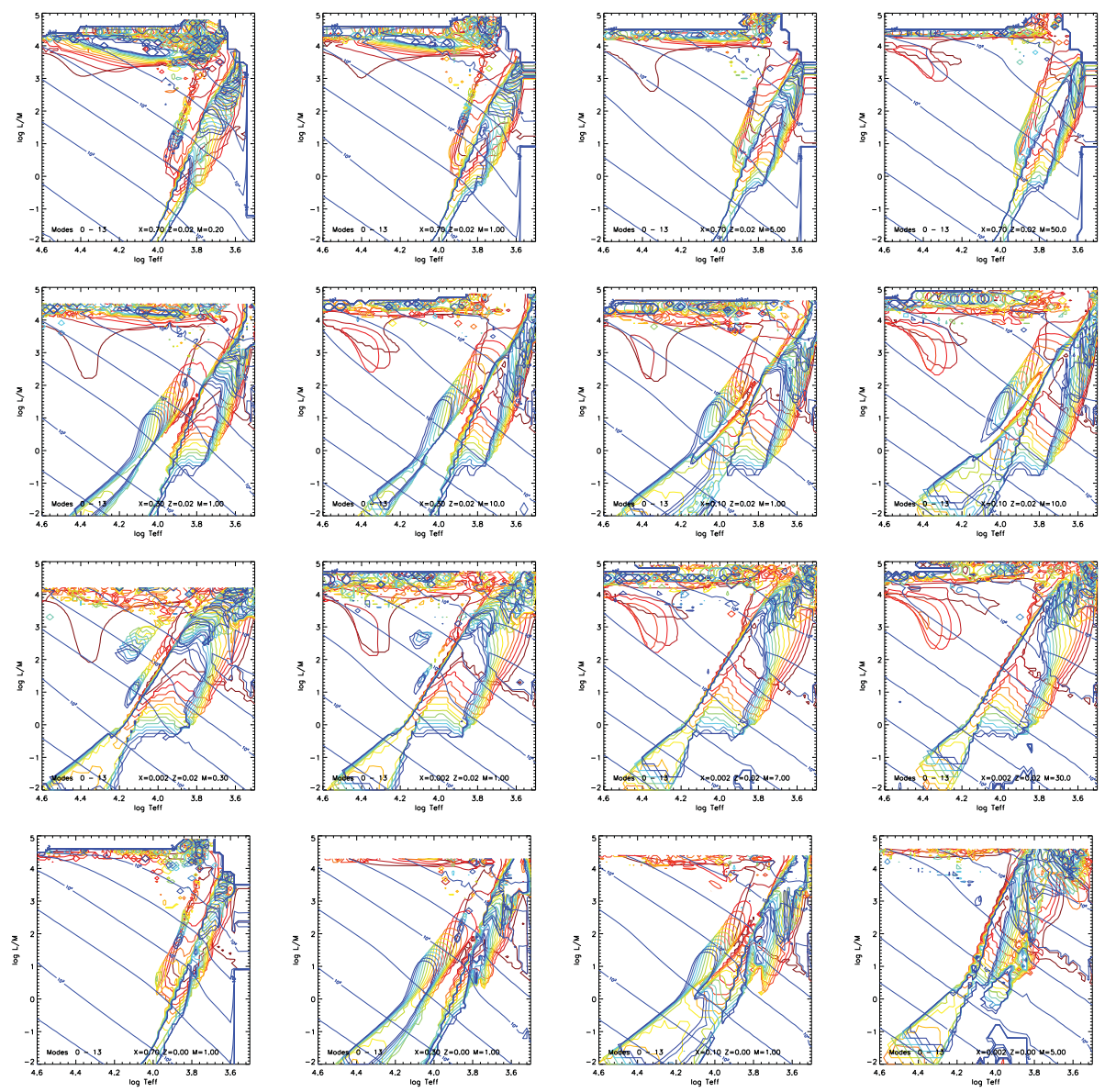

Figure 2. Unstable mode boundaries $n=0-10$. As Fig. 1, but showing the boundaries for individual radial modes as coloured contours, with the darkest red representing the boundary of the fundamental $(n=0)$ mode, with increasing higher orders $(n=1-10)$ represented progressively by colours of increasing frequency (orange, yellow, green, blue ....). Diagonal blue lines represent period contours for the radial fundamental mode.

The results are presented as contour plots representing the number of unstable modes as a function of $\left(T_{\text {eff }}, L / M\right)$ for each composition (Fig. 1). This provides an overall instability boundary since it includes pulsations in both low- and high-order modes. In some cases, envelope models with very high $L / M$ ratios and/or very low $T_{\text {eff }}$ were difficult to integrate due to very low densities in the equation of state; these appear as voids on the contour plots.

The instability boundaries for modes with $n=0,1$, or more nodes, i.e. the instability boundaries for the fundamental radial, and for the first and higher overtone pulsations are shown, also as a function of $T_{\text {eff }}, L / M$ and $X$ (Fig. 2).

Some models represent no known stars (e.g. high $M$ models with very low $L / M$ ). Nevertheless, exploring such models provides a systematic insight into the pulsation properties of stars in general.

The results vary only slowly with mass over the range considered: $0.2-50 \mathrm{M}_{\odot}$. 


\section{Unstable Radial Modes}

The use of the parameter $L / M$ exploits the fact that the radial pulsation properties of stellar envelopes vary slowly with mass over the range studied (cf. Fig. 1: top row). Several interesting features are apparent, amongst which we remark on the following:

At composition $X=0.70, Z=0.02$ we easily identify the classical instability strip where pulsations driven by $\mathrm{He}^{+}$are excited in the radial fundamental, first and second overtone modes ( $n=0,1,2$, Fig. 2 : top row).

Unstable modes at high $L / M$ are the strange modes (Saio et al. 1998), associated with $\alpha$ Cyg variables at high mass and $\mathrm{R} \mathrm{CrB}$ variables at low mass. The confusion in the mode boundary diagram (Fig. 3) is due to a breakdown in the strict 1-1 correspondence between mode eigenfrequency and node number for strange modes.

At the lowest $L / M$ only high-order modes are excited in the classical instability strip.

At $\log (L / M)>3$ and $\log T_{\text {eff }} \approx 4.4$, the weak instability finger is caused by iron-group opacities at temperatures $T \approx 2 \times 10^{5} \mathrm{~K}$ and identified with $\beta$ Cepheid variables. The shape of this finger is sensitive to mass.

At $X \leqslant 0.30$, the driving effect of $\mathrm{He}$ in the classical instability strip and $\mathrm{Fe}$ and $\mathrm{Ni}$ in the 'Z-bump' instability finger is increased, as hydrogen damping is reduced. This has several consequences, including:

The blue edge of the classical (Cepheid) instability strip becomes bluer.

The width of the high-order $\mathrm{He}^{+}$instability strip at low $L / M$ increases. At high mass, the region becomes fragmented. At low mass, the models suggest that radial modes in some DB white dwarfs may be unstable.

At $X=0.002$, an even bluer strip beomes unstable to high-order modes, and has been identified with pulsations observed in the pre-white dwarf J0247-25B (Jeffery \& Saio 2013).

High-order radial modes may be excited at other locations blueward of the classical instability strip in stars where the envelope hydrogen abundance is significantly depleted. For example, several He-rich low-mass white dwarfs (Gianninas et al. 2014) lie within a region where we find high-order p-modes to be unstable $\left(\log (L / M) \approx 1\right.$ and $\log T_{\text {eff }} \approx$ 4.15). Pulsations may also be excited in H-deficient horizontal-branch or low-mass postAGB stars with $\log (L / M) \approx 2-3$ and $\log T_{\text {eff }} \approx 4.0-4.2$.

\section{References}

Gianninas, A., Dufour, P., Kilic, M., Brown, W. R., Bergeron, P., \& Hermes, J. J. 2014, ApJ, 794,35

Hermes, J. J., Montgomery, M. H., Winget, D. E., Brown, W. R., Gianninas, A., Kilic, M., Kenyon, S. J., \& Bell, K. J. 2013, ApJ, 765, 102

Jeffery, C. S., 2008, Information Bulletin on Variable Stars, 5817, 1

Jeffery, C. S. \& Saio, H. 2006a, MNRAS, 371, 659

Jeffery, C. S. \& Saio, H. 2006b, MNRAS, 372, L48

Jeffery, C. S. \& Saio, H. 2007, MNRAS, 378, 379

Jeffery, C. S. \& Saio, H. 2013, MNRAS, 435, 885

Maxted, P. F. L., Serenelli, A. M., Miglio, A., Marsh, T. R., Heber, U., Dhillon, V. S., Littlefair, S., Copperwheat, S., Smalley, B., Breedt, E., \& Schaffenroth, V. 2013, Nature, 498, 463

Saio, H., Baker, N. H., \& Gautschy, A. 1998, MNRAS, 294, 622

Saio, H. \& Jeffery, C. S. 1988, ApJ, 328, 714

Saio, H., Winget, D. E., \& Robinson, E. L. 1983, ApJ, 265, 982 